\title{
Note on Laguerre Transform in Two Variables
}

\author{
Ajay Shukla, Ibrahim Abubaker Salehbhai \\ Department of Mathematics, S.V. National Institute of Technology, Surat, India \\ E-mail:ajayshukla2@rediffmail.com, ibrahimmaths@gmail.com \\ Received March 29, 2011; revised June 10, 2011; accepted June 10, 2011
}

\section{Abstract}

An attempt is made to investigate the some new properties of Laguerre transform in two variables [1].

Keywords: Laguerre Transform, Laguerre Polynomials in Two Variables, Laguerre Transform in Two Variables

\section{Introduction}

Debnath [2] introduced the Laguerre transform and derived some properties. He also discussed the applications in study of heat conduction [3] and to the oscillations of a very long and heavy chain with variable tension [4].

Glaeske generalized Laguerre transform of one variable as Laguerre-Pinney transformation [5], WienerLaguerre transformation [6] and derived its properties. Debnath et al. [7] reported all these work in their book.

Recently Shukla et al. [1] introduced the Laguerre Transform of $f(x, y)$ as

$$
\begin{aligned}
F_{n}(\alpha, \beta) & =\mathrm{L}\{f(x, y), x \rightarrow \alpha, y \rightarrow \beta, n\} \\
& =\int_{0}^{\infty} \int_{0}^{\infty} \mathrm{e}^{-(x+y)} x^{\alpha} y^{\beta} K_{n}^{(\alpha, \beta)}(x, y) f(x, y) \mathrm{d} x \mathrm{~d} y
\end{aligned}
$$

where $f(x, y)$ be a Riemann integrable function defined on the set $S=\mathbb{R}^{+} \times \mathbb{R}^{+}, \alpha>-1, \beta>-1, n$ is nonnegative integer and

$$
K_{n}^{(\alpha, \beta)}(x, y)=\sum_{r=0}^{n} \frac{(-x y)^{r}}{r !(-n)_{r}} L_{n-r}^{(\alpha+r, \beta+r)}(x, y)
$$

Ragab [8] introduced Laguerre polynomials of two variables $L_{n}^{(\alpha, \beta)}(x, y)$, which is defined as

$$
\begin{aligned}
& L_{n}^{(\alpha, \beta)}(x, y)=\frac{\Gamma(\alpha+n+1) \Gamma(\beta+n+1)}{n !} \\
& \cdot \sum_{k=0}^{n} \frac{L_{n-k}^{(\alpha)}(x)(-y)^{k}}{k ! \Gamma(\alpha+n-k+1) \Gamma(\beta+k+1)}
\end{aligned}
$$

Ragab [8] also obtained,

$$
K_{n}^{(\alpha, \beta)}(x, y)=L_{n}^{\alpha}(x) L_{n}^{\beta}(y)
$$

Therefore, the equivalent definition for the Laguerre Transform of $f(x, y)$ is

$$
\begin{aligned}
\mathrm{L}\{f(x, y)\} & =F_{n}(\alpha, \beta) \\
& =\int_{0}^{\infty} \int_{0}^{\infty} \mathrm{e}^{-(x+y)} x^{\alpha} y^{\beta} L_{n}^{\alpha}(x) L_{n}^{\beta}(y) f(x, y) \mathrm{d} x \mathrm{~d} y
\end{aligned}
$$

We also used following theorems based on Shukla et al. [1]:

Theorem 1: If $K_{n}^{(\alpha, \beta)}(x, y)$ is defined as (1.2), then

$$
\int_{0}^{\infty} \int_{0}^{\infty} \mathrm{e}^{-(x+y)} x^{\alpha} y^{\beta} K_{n}^{(\alpha, \beta)}(x, y) K_{m}^{(\alpha, \beta)}(x, y) \mathrm{d} x \mathrm{~d} y=\delta_{n} \delta_{m n}
$$

where $\delta_{m n}$ (Kronecker delta symbol) is defined as

$$
\begin{aligned}
& \delta_{m n}=\left\{\begin{array}{ll}
0, & m \neq n \\
1, & m=n
\end{array},\right. \\
& \delta_{n}=\frac{\Gamma(n+\alpha+1) \Gamma(n+\beta+1)}{(n !)^{2}}, \alpha>-1 \text { and } \beta>-1 .
\end{aligned}
$$

Srivastava and Manocha[9] reported following results:

$$
\begin{aligned}
& \sum_{m=0}^{\infty} \frac{m !(\lambda)_{m}}{(\alpha+1)_{m}(\beta+1)_{m}} L_{m}^{\alpha}(x) L_{m}^{\beta}(y) t^{m} \\
& =(1-t)^{-\lambda} \sum_{m=0}^{\infty} \frac{(\lambda)_{m}}{m !(\alpha+1)_{m}(\beta+1)_{m}}\left(\frac{x y t}{1-t}\right)^{m} \\
& \cdot \psi_{2}\left[\lambda+m ; \alpha+m+1, \beta+m+1 ; \frac{x t}{t-1}, \frac{y t}{t-1}\right],|t|<1
\end{aligned}
$$

where $\psi_{2}$ is defined as: 


$$
\psi_{2}\left[\alpha ; \beta, \beta^{\prime} ; x, y\right]=\sum_{m, n=0}^{\infty} \frac{(\alpha)_{m+n}}{(\beta)_{m}\left(\beta^{\prime}\right)_{n}} \frac{x^{m}}{m !} \frac{y^{n}}{n !}
$$

Equation (1.7) can be easily written as

$$
\begin{aligned}
& \sum_{m=0}^{\infty} \frac{m !(\lambda)_{m}}{(\alpha+1)_{m}(\beta+1)_{m}} L_{m}^{\alpha}(x) L_{m}^{\beta}(y) t^{m} \\
& =(1-t)^{-\lambda} \\
& \cdot F^{(3)}\left[\begin{array}{ccccccccccc}
\lambda:: & - & - & - & - & - & - & - & & & \\
& & & & & & & \xi & \eta & \zeta \\
-: & \alpha+1 ; & - & \beta+1: & \ldots & - & \ldots & & &
\end{array}\right]
\end{aligned}
$$

where

and

$$
\xi=\frac{x y t}{1-t}, \eta=\frac{-x t}{1-t}, \zeta=\frac{-y t}{1-t}
$$

We used following resultbased on Erdélyi et al. [10]:

$$
\begin{aligned}
& \sum_{m=0}^{\infty} \frac{m !(\lambda)_{m}}{(\alpha+1)_{m}(\beta+1)_{m}} L_{m}^{\alpha}(x) L_{m}^{\beta}(y) t^{m} \\
& =(1-t)^{-\lambda} \sum_{m=0}^{\infty} \frac{(\lambda)_{m}}{m !(\alpha+1)_{m}(\beta+1)_{m}}\left(\frac{x y t}{1-t}\right)^{m} \\
& \times_{1} F_{1}\left[\begin{array}{c}
\lambda+m ; \\
\alpha+m+1 ;
\end{array}\right]\left[\begin{array}{cc}
\lambda+m ; \\
t-1
\end{array}\right] F_{1}\left[\begin{array}{l}
t-1 \\
\beta+m+1 ;
\end{array}\right],|t|<1
\end{aligned}
$$

and following results (1.12 and 1.13) based on Rainville [11]:

$$
\begin{aligned}
& \int_{0}^{\infty} \mathrm{e}^{-x} x^{\alpha} L_{n}^{\alpha}(x) x^{k} \mathrm{~d} x=0 ; k=0,1,2, \cdots,(n-1) \\
& \sum_{m=0}^{k} \frac{m ! L_{m}^{\alpha}(x) L_{m}^{\alpha}(y)}{(1+\alpha)_{m}} \\
& =\frac{(k+1) !}{(1+\alpha)_{k}} \frac{L_{k+1}^{\alpha}(y) L_{k}^{\alpha}(x)-L_{k+1}^{\alpha}(x) L_{k}^{\alpha}(y)}{x-y}
\end{aligned}
$$

\section{Main Results}

In this section, some new properties of Laguerre Transforms in two variables [1] have been obtained.

Theorem 1: If

$$
\begin{aligned}
f(x, y)= & (1-t)^{-\lambda} \sum_{m=0}^{\infty} \frac{(\lambda)_{m}}{m !(\alpha+1)_{m}(\beta+1)_{m}}\left(\frac{x y t}{1-t}\right)^{m} \\
& \cdot \psi_{2}\left[\lambda+m ; \alpha+m+1, \beta+m+1 ; \frac{x t}{t-1}, \frac{y t}{t-1}\right],|t|<1
\end{aligned}
$$

then

$$
\mathrm{L}\{f(x, y), \alpha, \beta, n\}
$$

$$
=F_{n}(\alpha, \beta)=\frac{t^{n}(\lambda)_{n} \Gamma(\alpha+1) \Gamma(\beta+1)}{n !}
$$

Here $\psi_{2}$ is a function defined by (1.7).

Proof: Using (1.7) and (1.5), we have

$$
\begin{aligned}
F_{n}(\alpha, \beta)= & \int_{0}^{\infty} \int_{0}^{\infty} \mathrm{e}^{-(x+y)} x^{\alpha} y^{\beta} L_{n}^{\alpha}(x) L_{n}^{\alpha}(y) \\
& \cdot \sum_{m=0}^{\infty} \frac{m !(\lambda)_{m}}{(\alpha+1)_{m}(\beta+1)_{m}} L_{m}^{\alpha}(x) L_{m}^{\alpha}(y) t^{m} \mathrm{~d} x \mathrm{~d} y
\end{aligned}
$$

Further using (1.6), we arrived at

$$
F_{n}(\alpha, \beta)=\sum_{m=0}^{\infty} \frac{m !(\lambda)_{m}}{(\alpha+1)_{m}(\beta+1)_{m}} \delta_{n} \delta_{m n} t^{m}
$$

Using definition of $\delta_{m n}$, we get

$$
F_{n}(\alpha, \beta)=\frac{n !(\lambda)_{n}}{(\alpha+1)_{n}(\beta+1)_{n}} \delta_{n} t^{n}
$$

Using definition of $\delta_{n}$, we get

$$
F_{n}(\alpha, \beta)=\frac{t^{n}(\lambda)_{n} \Gamma(\alpha+1) \Gamma(\beta+1)}{n !}
$$

This completes the proof.

Using (1.9), we get

Corollary 1: If

$$
\begin{aligned}
& F^{(3)}\left[\begin{array}{llll}
(a):: & (b) ; & \left(b^{\prime}\right) ; \quad\left(b^{\prime \prime}\right):(c) ; \quad\left(c^{\prime}\right) ; \quad\left(c^{\prime \prime}\right) ; & \\
(e):: \quad(g) ; \quad\left(g^{\prime \prime}\right) ; \quad\left(g^{\prime \prime}\right):(h) ; \quad\left(h^{\prime}\right) ; \quad\left(h^{\prime \prime}\right) ; & x, \quad z
\end{array}\right] \\
& =\sum_{m, r, p=0}^{\infty} \frac{\left.\prod_{j=1}^{A}\left(a_{j}\right)_{m+r+p} \prod_{j=1}^{B}\left(b_{j}\right)_{m+r} \prod_{j=1}^{B^{\prime}}\left(b_{j}\right)_{r+p} \prod_{j+r+p}^{B^{\prime \prime}}\left(b_{j}^{\prime \prime}\right)_{p+m}\left(g_{j}\right)_{m+r} \prod_{j=1}^{G^{\prime}}\left(g_{j}^{\prime}\right)_{r+p} \prod_{j=1}^{G^{\prime \prime}}\left(g_{j}{ }_{j}^{\prime \prime}\right)_{p+m}\right)_{m} \prod_{j=1}^{C^{\prime}}\left(c_{j}^{\prime}\right)_{r} \prod_{j=1}^{C^{\prime \prime}}\left(c_{j}^{\prime \prime}\right)_{p}}{\prod_{j=1}^{H}\left(h_{j}\right)_{m} \prod_{j=1}^{H^{\prime}}\left(h_{j}^{\prime}\right)_{r} \prod_{j=1}^{H^{\prime \prime}}\left(h_{j}^{\prime \prime}\right)_{p}} \frac{x^{m}}{m !} \frac{y^{r}}{r !} \frac{z^{p}}{p !}
\end{aligned}
$$




$$
\begin{aligned}
& f(x, y)=(1-t)^{-\lambda} \\
& \cdot F^{(3)}\left[\begin{array}{cccccccccc}
\lambda \because: & - & - & - & -; & -; & -; & & \\
& & & & & & & \xi & \eta & \zeta \\
-\because & \alpha+1 ; & -; & \beta+1: & - & -; & -; & & &
\end{array}\right]
\end{aligned}
$$

where $\xi=\frac{x y t}{1-t}, \eta=\frac{-x t}{1-t}, \zeta=\frac{-y t}{1-t}$

then

$$
\begin{aligned}
& \mathrm{L}\{f(x, y), \alpha, \beta, n\} \\
& =F_{n}(\alpha, \beta)=\frac{t^{n}(\lambda)_{n} \Gamma(\alpha+1) \Gamma(\beta+1)}{n !}
\end{aligned}
$$

Here $F^{3}$ is a function defined by (1.10).

Also, using (1.11) we have

\section{Corollary 2:}

If

$$
\begin{aligned}
f(x, y) & =(1-t)^{-\lambda} \sum_{m=0}^{\infty} \frac{(\lambda)_{m}}{m !(\alpha+1)_{m}(\beta+1)_{m}}\left(\frac{x y t}{1-t}\right)^{m} \\
& \times{ }_{1} F_{1}\left[\begin{array}{c}
\lambda+m ; \\
\alpha+m+1 ;
\end{array}\right]{ }_{1} F_{1}\left[\begin{array}{c}
\lambda+m ; \\
\beta+m+1 ;
\end{array}\right],
\end{aligned}
$$

$$
(|t|<1)
$$

then,

$$
\begin{aligned}
& \mathrm{L}\{f(x, y), \alpha, \beta, n\} \\
& =F_{n}(\alpha, \beta)=\frac{t^{n}(\lambda)_{n} \Gamma(\alpha+1) \Gamma(\beta+1)}{n !}
\end{aligned}
$$

Theorem 2: If $f(x, y)=x^{k} y^{l}$, where $k$ and $l$ are positive numbers such that $k=0,1,2, \cdots,(n-1)$ or $l=0,1,2, \cdots,(n-1)$ then

$$
\mathrm{L}\{f(x, y)\}=0
$$

Using (1.12) we can obtain (2.4).

\section{Theorem 3:}

If

$$
f(x, y)=\frac{(k+1) !}{(1+\alpha)_{k}} \frac{L_{k+1}^{\alpha}(y) L_{k}^{\alpha}(x)-L_{k+1}^{\alpha}(x) L_{k}^{\alpha}(y)}{x-y}
$$

and $F_{n}(\alpha, \beta)=\mathrm{L}\{f(x, y), \alpha, \beta, n\}$ then,

$$
F_{n}(\alpha, \alpha)=\frac{\Gamma(\alpha+1) \Gamma(n+\alpha+1)}{n !}
$$

Proof: Using (1.13) and (1.5), we have

$$
\begin{aligned}
& F_{n}(\alpha, \alpha) \\
& =\int_{0}^{\infty} \int_{0}^{\infty} \mathrm{e}^{-(x+y)} x^{\alpha} y^{\alpha} K_{n}^{(\alpha, \alpha)}(x, y) \sum_{m=0}^{k} \frac{m ! L_{m}^{\alpha}(x) L_{m}^{\alpha}(y)}{(1+\alpha)_{m}} \mathrm{~d} x \mathrm{~d} y
\end{aligned}
$$

Further using (1.6), we arrived at

$$
F_{n}(\alpha, \alpha)=\sum_{m=0}^{k} \frac{m !}{(\alpha+1)_{m}} \delta_{n} \delta_{m n}
$$

Using definition of $\delta_{m n}$, we get

$$
F_{n}(\alpha, \alpha)=\frac{n !}{(\alpha+1)_{n}} \delta_{n}
$$

Using definition of $\delta_{n}$, we get (2.5).

\section{References}

[1] A. K. Shukla, I. A. Salehbhai and J. C. Prajapati, "On the Laguerre Transform in Two Variables,” Integral Transforms and Special Functions, Vol. 20, No. 6, 2009, pp. 459-470. doi:10.1080/10652460802645818

[2] L. Debnath, “On Laguerre Transform,” Bulletin of Calcutta Mathematical Society, Vol. 55, 1960, pp. 69-77.

[3] L. Debnath, “Application of Laguerre Transform to Heat Conduction Problem,” Annali dell' University di Ferrara, Sezione VII-ScienzeMathematiche, Vol. X, 1962, pp. 1719.

[4] L. Debnath, "Application of Laguerre Transform to the Problem of Oscillations of a very Long and Heavy Chain,” Annali dell' Univ. di Ferrara, Sezione VII-ScienzeMathe-matiche, Vol. IX, No. 1, 1961, pp. 149-151.

[5] H. J. Glaeske, "Die Laguerre-Pinney Transformation," Aequationesmathematicae, Vol. 22, No. 1, 1981, pp. 7385. doi:10.1007/BF02190163

[6] H. J. Glaeske, "On the Wiener-Laguerre Transformation,” Riview T'ec Ing University Zulia, Vol. 9, No. 1, 1986, pp. 27-34.

[7] L. Debnath and D. Bhatta, "Integral Transforms and Their Applications,” Chapman \& Hall, New York, 2007.

[8] S. F. Ragab, "On Laguerre Polynomials of Two Variables,” Bulletin of Calcutta Mathematical Society, Vol. 83, 1991, pp. 253-262.

[9] H. M. Srivastava and H. L. Manocha, “A Treatise on Generating Functions,” John Wiley and Sons, New York, 1984.

[10] A. Erdélyi, “Higher Transcendental Functions,” Vol. 2, McGraw-Hill, New York, 1953.

[11] E. D. Rainville, “Special Functions,” Macmillan, New York, 1960. 